\title{
Albumin-bilirubin grade-based nomogram of the BCLC system for personalized prognostic prediction in hepatocellular carcinoma
}

\author{
Shu-Yein Ho ${ }^{1,2}$ | Chia-Yang Hsu ${ }^{2,3}$ | Po-Hong Liu' ${ }^{2,4}$ | Cheng-Yuan Hsia ${ }^{2,5}$ | \\ Hao-Jan Lei ${ }^{2,5}$ | Yi-Hsiang Huang, ${ }^{1,2,6}$ (D) | Chih-Chieh Ko ${ }^{1,2}$ | Chien-Wei Su', (D) | \\ Rheun-Chuan Lee ${ }^{2,7}$ | Ming-Chih Hou ${ }^{1,2}$ | Teh-la Huo ${ }^{2,8,9}$
}

${ }^{1}$ Department of Medicine, Taipei Veterans General Hospital, Taipei, Taiwan

${ }^{2}$ Faculty of Medicine, National Yang-Ming University School of Medicine, Taipei,

Taiwan

${ }^{3}$ Division of Gastroenterology and Hepatology, University of Michigan, Ann Arbor, MI, USA

${ }^{4}$ Department of Internal Medicine, University of Texas Southwestern Medical Center, Dallas, TX, USA

${ }^{5}$ Department of Surgery, Taipei Veterans General Hospital, Taipei, Taiwan

${ }^{6}$ Institute of Clinical Medicine, National Yang-Ming University School of Medicine, Taipei, Taiwan

${ }^{7}$ Department of Radiology, Taipei Veterans General Hospital, Taipei, Taiwan

${ }^{8}$ Department of Medical Research, Taipei Veterans General Hospital, Taipei, Taiwan

${ }^{9}$ Institute of Pharmacology, National YangMing University School of Medicine, Taipei, Taiwan

\section{Correspondence}

Teh-la Huo, Professor of Medicine, Department of Medical Research, Taipei Veterans General Hospital, No. 201, Sec. 2 , Shipai Rd., Taipei 11217, Taiwan.

Email: tihuo@vghtpe.gov.tw

Funding information

Taipei Veterans General Hospital, Grant/ Award Number: V107A-008, VN108-05, V108A-002 and V108C-008

Handling Editor: Juan Abraldes

\begin{abstract}
Background \& Aims: The prognostic accuracy of individual hepatocellular carcinoma (HCC) patient in each Barcelona Clinic Liver Cancer (BCLC) stage is unclear. We aimed to develop and validate an albumin-bilirubin (ALBI) grade-based nomogram of BCLC to estimate survival for individual HCC patient.

Methods: Between 2002 and 2016, 3690 patients with newly diagnosed HCC were prospectively enrolled and retrospectively analysed. Patients were randomly split into derivation and validation cohort by $1: 1$ ratio. Multivariate Cox proportional hazards model was used to generate the nomogram from tumour burden, ALBI grade and performance status (PS). The concordance index and calibration plot were determined to evaluate the performance of this nomogram.

Results: Beta coefficients from the Cox model were used to assign nomogram points to different degrees of tumour burden, ALBI grade and PS. The scores of the nomogram ranged from 0 to 24 , and were used to predict 3- and 5-year patient survival. The concordance index of this nomogram was 0.77 (95\% confidence interval $[\mathrm{Cl}]$ : 0.71-0.81) in the derivation cohort and 0.76 (95\% Cl: 0.71-0.81) in the validation cohort. The calibration plots to predict both 3- and 5-year survival rate well matched with the 45-degree ideal line for both cohorts, except for ALBI-based BCLC stage 0 in the validation cohort.

Conclusions: The proposed ALBI-based nomogram of BCLC system is a simple and feasible strategy in the precision medicine era. Our data indicate it is a straightforward and user-friendly prognostic tool to estimate the survival of individual HCC patient except for very early stage patients.
\end{abstract}

KEYWORDS

ALBI grade, BCLC, nomogram 


\section{1 | INTRODUCTION}

Hepatocellular carcinoma ( $\mathrm{HCC}$ ) is the fourth common cause of cancer-related death worldwide in $2018 .{ }^{1}$ Despite improvement in diagnosis and treatments, the prognosis generally remains poor. Staging systems have been used to predict the prognosis in cancer patients. Up to now, at least 11 staging systems were proposed for HCC to assess patient survival. ${ }^{2}$ According to the American Association for the Study of Liver Diseases and European Association for the Study of the Liver practice guidelines, the Barcelona Clinic Liver Cancer (BCLC) staging system is the currently recommended staging system. ${ }^{3,4}$ Its advantages are clear stratification of HCC patients into different stages and treatment guidance.

The BCLC system incorporates three major prognostic parameters: tumour burden, liver functional reserve and performance status (PS). ${ }^{3,4}$ The traditional Child-Turcotte-Pugh (CTP) classification, based on five clinical and laboratory data, is employed to assess liver function in the BCLC and many other staging systems. ${ }^{2}$ However, there are some shortcomings because it contains subjective variables such as ascites and hepatic encephalopathy which often make clinical interpretation inconsistent. The albumin-bilirubin (ALBI) grade, a simple and objective tool that only includes serum albumin and bilirubin level, has been proposed to assess liver functional reserve in HCC patients. ${ }^{5,6}$ Several recent studies subsequently validated the ALBI grade as an appropriate or better model to evaluate liver reserve than the CTP classification. ${ }^{7-9}$

A nomogram is a graphic presentation of a multivariable model that generates numerical probabilities of an event. ${ }^{10,11}$ Because of its convenience in clinical application, several nomograms have been developed to predict the prognosis in various cancers. ${ }^{12-16} \mathrm{~A}$ nomogram can provide an individualized, evidence-based, highly accurate risk estimation. With the help of nomogram, physicians can accurately estimate the risk of a specific event at an individual level which is beneficial in clinical decision-making. In this study, we have constructed and validated an ALBI-based nomogram of the BCLC system to predict individual survival probability in a large HCC patient cohort.

\section{2 | METHODS}

\section{1 | Patients}

In a 14-year period between February 2002 and December 2016, a total of 3690 patients with newly diagnosed $\mathrm{HCC}$ were prospectively enrolled and retrospectively analysed in Taipei Veterans General Hospital, a 3000-bed tertiary referral medical centre in northern Taiwan. The baseline demographics, clinical information, PS, severity of liver dysfunction, serum biochemistry and cancer staging were comprehensively recorded at the time of diagnosis. Patients were randomly split into derivation and validation cohort by $1: 1$ ratio. Survival of the enrolled patients was inspected every 3-4 months from the hospital records and was cross-referenced with the database of Taiwan National Cancer Registry. The study was approved

\section{Key points}

- The albumin-bilirubin (ALBI) grade is a simple, objective and evidence-based assessment of liver functional reserve in patients with hepatocellular carcinoma (HCC).

- The integration of ALBI grade into Barcelona Clinic Liver Cancer (BCLC) system was proposed, but the prognosis greatly varied in each stage.

- The ALBI grade-based nomogram of BCLC was developed in this study and showed good performance in prognostic prediction.

- This nomogram can accurately estimate the survival for individual HCC patient at 3 and 5 years except for very early stage patients.

by the institutional review board (IRB) of the hospital and complies with the standards of Declaration of Helsinki. Patient information was de-identified prior to analysis. Informed consent was waived by the IRB because of the retrospective nature of this study.

\section{2 | Diagnosis and definition}

The diagnosis of HCC was confirmed by distinctive pattern of hyperenhancement in the arterial phase and washout in venous or delayed phases on multiphasic dynamic contrast-enhanced computer tomography (CT) or magnetic resonance imaging (MRI), or confirmed by pathology if there was no typical hallmark of HCC on imaging. ${ }^{3,4,17}$ Staging of HCC was established when the diagnosis was confirmed. Patients who were seropositive for hepatitis B surface ( $\mathrm{HBsAg}$ ), seronegative for antibody against hepatitis $\mathrm{C}$ virus (anti-HCV) and no history of alcoholism were classified as HBV-related HCC. HCV-related $\mathrm{HCC}$ was defined as seropositive for anti-HCV, seronegative for HBsAg and without a history of alcoholism. ${ }^{18}$ Patients who drink at least $40 \mathrm{~g}$ alcohol daily for 5 years or more were considered as alcoholics. ${ }^{19}$ Vascular invasion was defined as radiological evidence of tumour invasion to intrahepatic vasculatures, portal trunk or abdominal great vessels. ${ }^{20}$ Lymph nodes metastasis was diagnosed by radiographic evidence of enlarged nodes $(>1 \mathrm{~cm})$ or on histological confirmation. Distant metastasis such as lung, lymph node and bone was diagnosed by $\mathrm{CT}$, MRI or bone scan. ${ }^{21}$ The ALBI score was calculated according to the following equation $=0.66 \times \mathrm{log}$ ${ }_{10}$ bilirubin - $0.085 \times$ albumin. ALBI grades were defined as ALBI grade 1 (score $\leq-2.60$ ), ALBI grade 2 (score $>-2.60$ and $\leq-1.39$ ) and ALBI grade 3 (score $>-1.39$ ). During calculation, serum bilirubin was expressed in $\mu \mathrm{mol} / \mathrm{L}$ and serum albumin level was expressed in $\mathrm{g} / \mathrm{L} .^{5,6,22}$ Tumour burden was grouped into five different grades as defined previously ${ }^{23}$ : tumour burden grade 0 (single tumour less than $2 \mathrm{~cm}$, without vascular invasion or distant metastasis), tumour burden grade 1 (single tumour size between $2 \mathrm{~cm}$ and $5 \mathrm{~cm}$ and three tumour nodules equal or less than $3 \mathrm{~cm}$, no vascular invasion or distant metastasis), tumour burden grade 2 (multiple tumours or 
tumour size greater than $5 \mathrm{~cm}$, without vascular invasion or distant metastasis), tumour burden grade 3 (tumour of any size with vascular invasion and distant metastasis). PS was assessed by using the Eastern Cooperative Oncology Group performance scale: 0 (asymptomatic) to 4 (confined to bed). ${ }^{24}$

\section{3 | ALBI grade-based BCLC (ALBI-BCLC) staging system}

Patients with tumour burden grade 1, ALBI grade 1 , PS 0 were defined as ALBI-based BCLC stage 0 , and patients with tumour burden grade 2 , ALBI grade 1 or 2 , PS 0 were grouped as ALBI-based BCLC stage $A$. ALBI-based BCLC stage $B$ was defined as tumour burden grade $2, A L B I$ grade 1 or 2 , PS 0 , and ALBI-based BCLC stage $C$ was defined as tumour grade 3, ALBI grade 1 or 2 and PS 1 or 2. Patients with any tumour burden grade, ALBI grade 3 , PS 3 or 4 were classified as ALBI-based BCLC stage D (Table 1).

\section{4 | Treatments}

After the diagnosis was confirmed, patients were reviewed at our multidisciplinary HCC team for treatment recommendation. Shared decision-making regarding treatment modalities was made by patients and physicians after individualized counselling. Written informed consent was obtained prior to any definite treatment. Surgical resection, radiofrequency ablation (RFA) and transarterial chemoembolization were performed by the standard procedures as described previously. ${ }^{25-27}$

\section{5 | Statistics}

The Mann-Whitney $U$ test was used to compare continuous variables between two groups. Category data were assessed by the Chi-square test and two-tailed Fisher exact test. The comparison of survival distribution was performed by the Kaplan-Meier method with log-rank test. Multivariate Cox proportional hazards model was used to determine the BETAs and hazard ratios of the prognostic factors including tumour burden, ALBI grade and PS.

TAB LE 1 Albumin-bilirubin (ALBI) grade-based Barcelona Clinical Liver Cancer (BCLC) staging system

\begin{tabular}{llllll} 
ALBI-BCLC & 0 & A & B & C & D \\
$\begin{array}{l}\text { Tumour burden } \\
\text { grade }\end{array}$ & 0 & 1 & 2 & 3 & Any \\
$\begin{array}{l}\text { ALBI grade } \\
\begin{array}{l}\text { Performance } \\
\text { status }\end{array}\end{array}$ & 0 & 0 & 0 & $1-2$ & $3-4$ \\
\hline
\end{tabular}

Note: Tumour burden grade 0: single tumour less than $2 \mathrm{~cm}$, without vascular invasion or distant metastasis, tumour burden grade 1: single tumour size between $2 \mathrm{~cm}$ and $5 \mathrm{~cm}$ or three tumour nodules equal or less than $3 \mathrm{~cm}$, no vascular invasion or distant metastasis, tumour burden grade 2: multiple tumours or single tumour greater than $5 \mathrm{~cm}$, without vascular invasion or distant metastasis, tumour burden grade 3 : tumour of any size with vascular invasion or distant metastasis.
Prognostic discrimination of the nomogram model was examined by the concordance index, which provides the probability that for two randomly selected patients, when one patient has an event (death) after the other, this patient has a better outcome prediction as determined by the nomogram. ${ }^{28}$ The calibration plot was generated by comparing the survival distribution observed by the Kaplan-Meier method with the means of nomogram-predicted survival after grouping patients into five stages based on ALBI-based BCLC system. All statistical analyses were performed using IBM SPSS Statistics for Windows, Version 21.0 (IBM Corp., Armonk, NY, USA) and SAS 9.4 (SAS Institute Inc). A $P$ value $<.05$ was considered statistically significant.

\section{3 | RESULTS}

\section{1 | Baseline characteristics of patients}

The baseline characteristics and clinical information of the study patients are summarized in Table 2. A total of 3690 patients were included for analysis. Their mean age was 65 years and $76 \%$ of patients were male. The most common aetiology was hepatitis $B$ (40\%) and the majority of patients was CTP class A (76\%). Patient stratification according to the ALBI grade was grade 1 in $38 \%$, grade 2 in $52 \%$ and grade 3 in $10 \%$ of patients. Regarding the tumour status, 2354 (64\%) patients presented with single tumour, and 1619 (44\%) patients had tumour size greater than $5 \mathrm{~cm}$. In addition, vascular invasion or metastasis was documented in 1034 (28\%) of patients, and 2186 (59\%) of patients were classified as PS 0. We re-staged the entire cohort according to the ALBI gradebased BCLC (ALBI-BCLC) system. A total 181 (5\%), 981 (26\%), 621 (17\%), 1319 (36\%) and 588 (16\%) patients were categorized into ALBI-BCLC stage $0, A, B, C$ and $D$ respectively. There were no significant baseline differences between the derivation and validation cohort (all $P>$.05).

\section{2 | Treatment and survival analysis}

The primary treatment modalities for both derivation and validation cohort according to ALBI-BCLC stage are shown in Table 3. For patients with ALBI-BCLC stage $0, A, B, C$ and $D$, there were $90 \%, 79 \%, 44 \%, 33 \%$ and $15 \%$ of patients in each respective category who underwent curative treatments. The median survival of the entire cohort was 31 months $(95 \%$ confidence interval $[\mathrm{Cl}]$ : 28.4-34.6 months). The selection of treatment in relation to ALBIBCLC stage and mortality is described in Table 2. There were no significant differences between the derivation and validation cohort according to treatments (all $P>.05$ ).

The 1-, 3- and 5-year survival rates for the entire cohort were $66 \%, 47 \%$ and $35 \%$ respectively. In the derivation cohort, there was a significant survival difference between different stages of the ALBI-BCLC system $(P<.001$, Figure $1 A)$. Similarly, early stage patients of ALBI-BCLC had better survival than patients at advanced cancer stage in the validation cohort $(P<.001$, Figure 1B). 
TAB LE 2 Baseline characteristics of the derivation and validation cohort

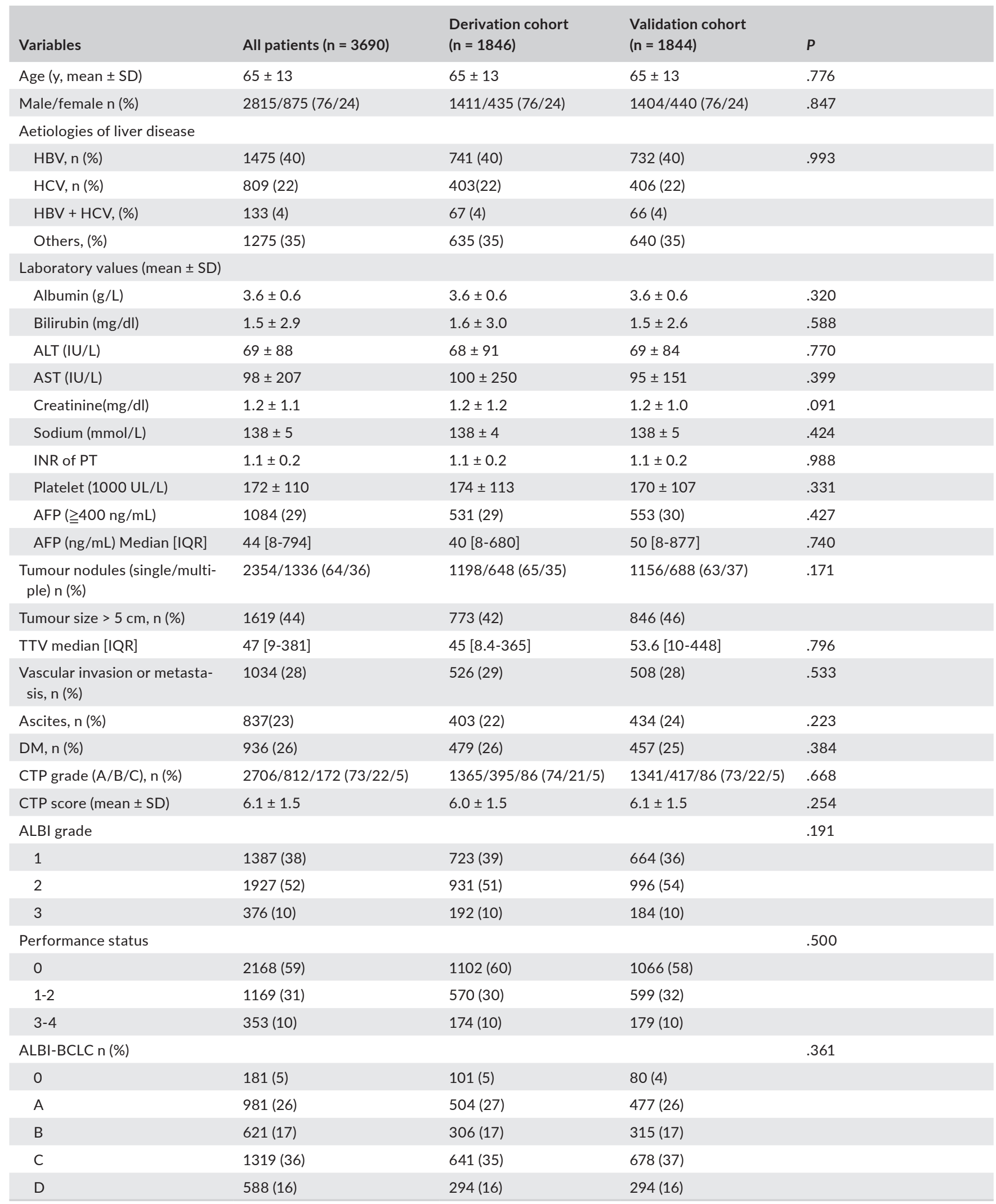

Abbreviations: ALBI, albumin-bilirubin; ALT, alanine aminotransferase; AST, aspartate aminotransferase; AFP, alpha-fetoprotein; CTP, Child-TurcottePugh; DM, diabetes mellitus; HBV, hepatitis B virus; HCV, hepatitis C virus; INR of PT, international normalized ratio of prothrombin time; MELD, model of end-stage liver disease; TACE, transarterial chemoembolization. 
TABLE 3 Treatment allocation

\begin{tabular}{|c|c|c|c|}
\hline $\begin{array}{l}\text { Number of patients, median and range of } \\
\text { follow-up (mo) and number of confirmed } \\
\text { mortality }\end{array}$ & $\begin{array}{l}\text { Derivation co- } \\
\text { hort }(n=1846)\end{array}$ & $\begin{array}{l}\text { Validation cohort } \\
(n=1844)\end{array}$ & $P$ \\
\hline $\begin{array}{l}\text { ALBI-based BCLC stage } 0(n=181, \\
\text { median }=63[1-192], 57)\end{array}$ & $101(5)$ & $80(4)$ & .842 \\
\hline Resection & 42 & 37 & \\
\hline Ablation & 48 & 35 & \\
\hline Transplantation & 1 & 1 & \\
\hline TACE & 9 & 5 & \\
\hline Targeted therapy & 0 & 0 & \\
\hline Radiotherapy & 0 & 0 & \\
\hline Supportive care & 1 & 2 & \\
\hline $\begin{array}{l}\text { ALBI-based BCLC stage A }(n=981, \\
\text { median }=47[1-242], 485)\end{array}$ & $504(27)$ & $477(26)$ & .165 \\
\hline Resection & 229 & 191 & \\
\hline Ablation & 168 & 181 & \\
\hline Transplantation & 1 & 2 & \\
\hline TACE & 102 & 93 & \\
\hline Targeted therapy & 0 & 0 & \\
\hline Radiotherapy & 0 & 0 & \\
\hline Supportive care & 4 & 10 & \\
\hline $\begin{array}{l}\text { ALBI-based BCLC stage B }(n=621, \\
\text { median }=28[1-177], 410)\end{array}$ & 306 (17) & 315 (17) & .959 \\
\hline Resection & 125 & 123 & \\
\hline Ablation & 19 & 15 & \\
\hline Transplantation & 1 & 1 & \\
\hline TACE & 141 & 151 & \\
\hline Targeted therapy & 6 & 6 & \\
\hline Radiotherapy & 1 & 1 & \\
\hline Supportive care & 13 & 18 & \\
\hline $\begin{array}{l}\text { ALBI-based BCLC stage } C(n=1319, \\
\text { median }=10[1-165], 955)\end{array}$ & $641(35)$ & $678(37)$ & .734 \\
\hline Resection & 133 & 163 & \\
\hline Ablation & 66 & 78 & \\
\hline Transplantation & 1 & 2 & \\
\hline TACE & 226 & 226 & \\
\hline Targeted therapy & 110 & 103 & \\
\hline Radiotherapy & 9 & 9 & \\
\hline Supportive care & 96 & 97 & \\
\hline $\begin{array}{l}\text { ALBI-based BCLC stage } D(n=588, \\
\text { median }=2[1-185], 504)\end{array}$ & $294(16)$ & $294(16)$ & .252 \\
\hline Resection & 11 & 15 & \\
\hline Ablation & 31 & 31 & \\
\hline Transplantation & 5 & 3 & \\
\hline TACE & 42 & 52 & \\
\hline Targeted therapy & 31 & 41 & \\
\hline Radiotherapy & 4 & 9 & \\
\hline Supportive care & 170 & 143 & \\
\hline
\end{tabular}

Abbreviations: ALBI, albumin-bilirubin; BCLC, Barcelona Clinic Liver Cancer; TACE, transarterial chemoembolization. 
(A)

$$
\text { Derivation cohort }(n=1846)
$$

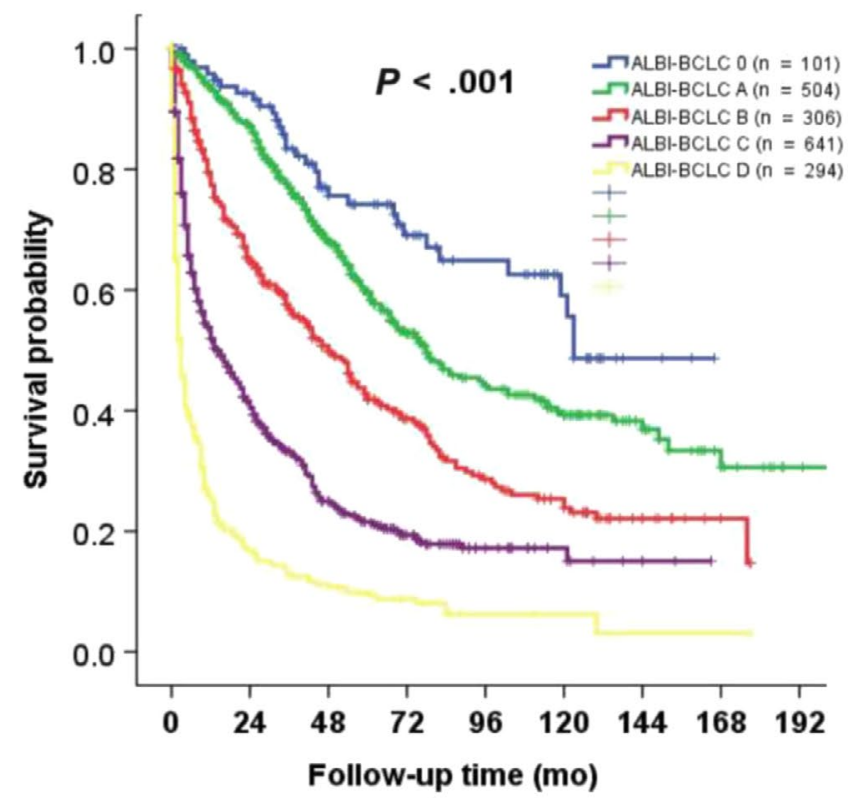

(B)

$$
\text { Validation cohort }(n=1844)
$$

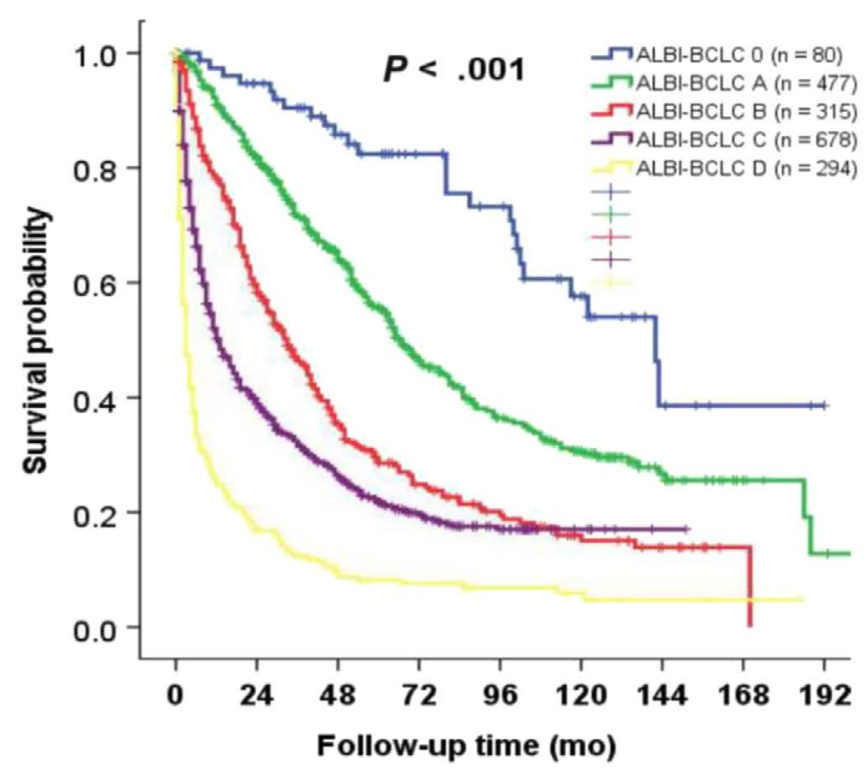

FIG URE 1 A, Comparison of survival distribution according to albumin-bilirubin (ALBI) grade-based Barcelona Clinic Liver Cancer (BCLC) staging system in the derivation cohort. B, Comparison of survival distribution according to ALBI grade-based BCLC system in the validation cohort. There is significant survival difference between different stages in both derivation and validation cohort $(P<.001)$

\begin{tabular}{|c|c|c|c|c|c|}
\hline & BETA & $\begin{array}{l}\text { BETA } \times 10 / \text { BETA of } \\
\text { tumour burden grade } 3\end{array}$ & $P$ & Hazard ratio (HR) & $\begin{array}{l}95 \% \text { confidence } \\
\text { interval }\end{array}$ \\
\hline $\begin{array}{l}\text { Tumour burden } \\
\text { grade } 0\end{array}$ & 0 & & & 1 & \\
\hline $\begin{array}{l}\text { Tumour burden } \\
\text { grade } 1\end{array}$ & 0.260 & 1.64 & .023 & 1.297 & $1.036-1.623$ \\
\hline $\begin{array}{l}\text { Tumour burden } \\
\text { grade } 2\end{array}$ & 0.718 & 4.54 & $<.001$ & 2.051 & $1.642-2.561$ \\
\hline $\begin{array}{l}\text { Tumour burden } \\
\text { grade } 3\end{array}$ & 1.579 & 10 & $<.001$ & 4.851 & $3.874-6.073$ \\
\hline ALBI grade 1 & 0 & & & 1 & \\
\hline ALBI grade 2 & 0.665 & 4.21 & $<.001$ & 1.944 & $1.697-2.227$ \\
\hline ALBI grade 3 & 1.051 & 6.66 & $<.001$ & 2.862 & $2.323-3.525$ \\
\hline $\begin{array}{l}\text { Performance status } \\
3-4\end{array}$ & 1.084 & 6.87 & $<.001$ & 2.957 & $2.417-3.618$ \\
\hline
\end{tabular}

TAB LE 4 Multivariate survival analysis of patients in derivation cohort $(n=1846)$

Abbreviation: ALBI, albumin-bilirubin.

\subsection{Construction of nomogram in the derivation cohort}

The three major prognostic predictors, ALBI grade, tumour burden and PS, were introduced into multivariate Cox model in the derivation cohort to generate the ALBI-BCLC nomogram (Table 4). With tumour burden grade $0, A L B I$ grade 1 and PS 0 as baseline reference, the impact of prognostic predictors was calculated for tumour burden grade 1 (BETA $=0.260, P=.023)$, tumour burden grade 2 (BETA = 0.718, $P<.001)$, tumour burden grade 3 (BETA $=1.579, P<.001)$, ALBI grade 2 (BETA $=0.665, P<.001$ ), ALBI grade 3 (BETA $=1.051, P<.001)$, PS 1-2 (BETA $=0.439, P<.001)$, PS 3-4 (BETA = 1.084, $P<.001)$. 
FIGURE 2 Albumin-bilirubin (ALBI) grade-based nomogram of Barcelona Clinic Liver Cancer (BCLC) to predict 3- and 5-y survival of hepatocellular carcinoma patients in the derivation cohort. The nomogram is used by adding up the points identified on the scale of the three prognostic predictors. The ranges of nomogram points for patients with different ALBI-based BCLC stages are shown in the figure. The total points project downward to obtain the estimated 3- and 5-y survival

Point

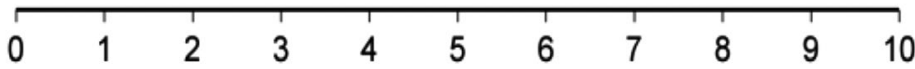

ALBI grade

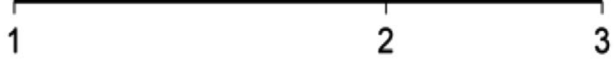

Performance

status

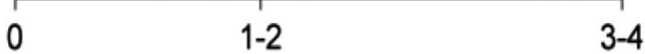

Tumor burden

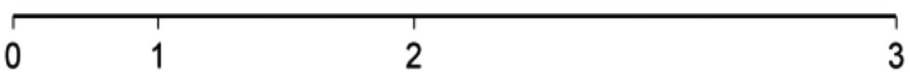

Total points

ALBI-BCLC

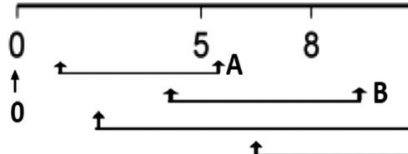

13

$17 \quad 19$

24

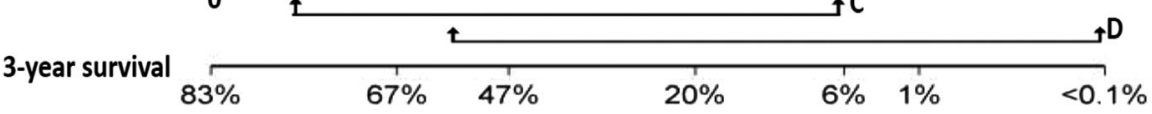

5-year survival $76 \%$

$54 \% \quad 32 \%$

$8 \%$

$1 \%<0.1 \%$

$<0.1 \%$
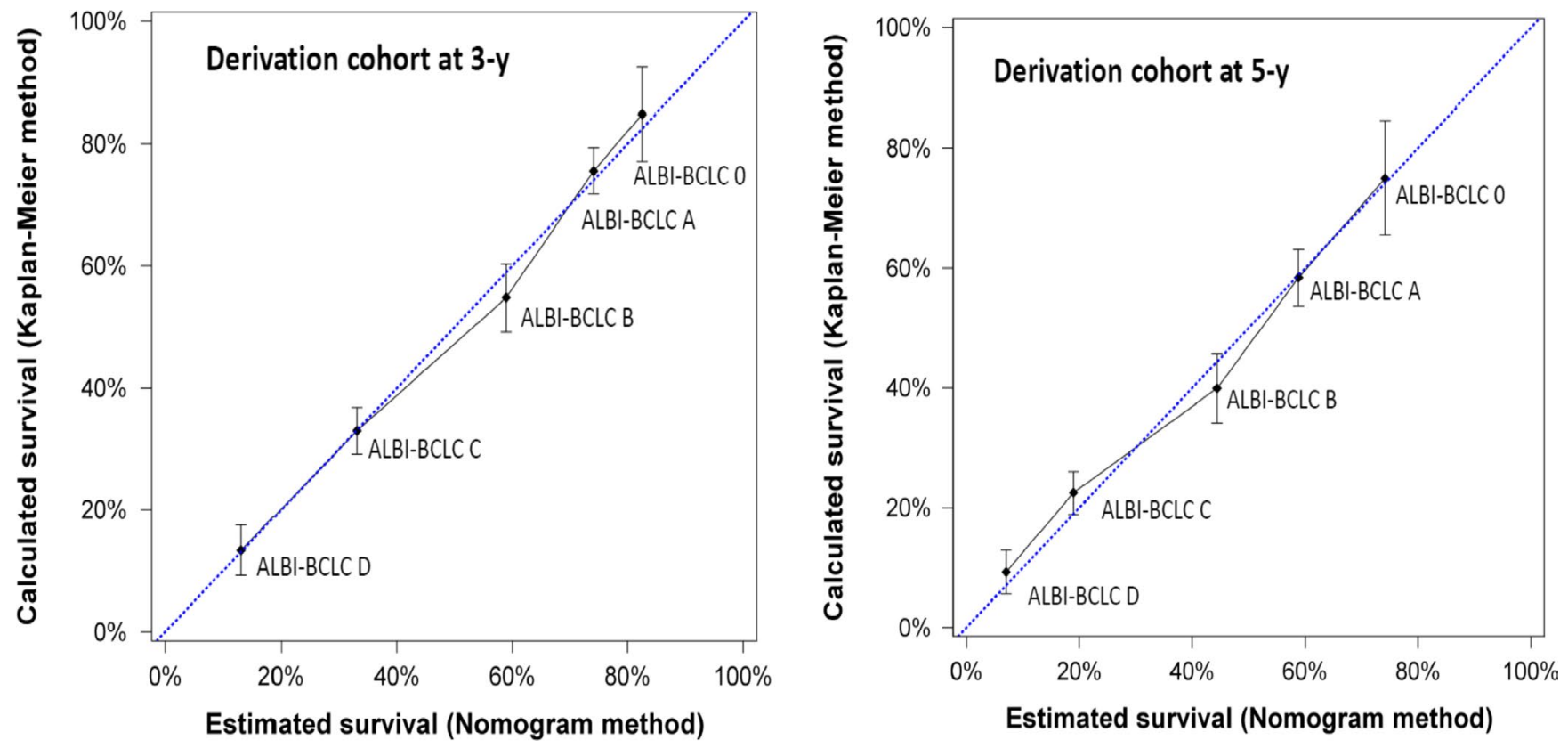

FIGURE 3 The calibration plots of the nomogram in the derivation cohort for 3- and 5-y survival prediction. The X-axis represents the nomogram-predicted survival and the $Y$-axis shows the mean survival and $95 \%$ confidence interval observed by the Kaplan-Meier method. By dividing patients into five groups based on the nomogram points, the calibration line fits along with the ideal 45-degree reference for both 3- and 5-y survival prediction

The nomogram was constructed using BETA coefficients from the Cox model. Tumour burden grade 3 had the highest impact in this model and was given 10 points in this nomogram. Sequentially, by using the ratios of BETAs between other prognostic factors and tumour burden grade $3,1.64$ (calculated as 0.260 divided by 1.579 and times 10), 4.54, 4.21, 6.66, 2.78 and 6.87 points were assigned to tumour burden grade 1, tumour burden grade 2, ALBI grade 2, ALBI grade 3, PS 1-2 and PS 3-4 respectively. Each patient had one individualized score from 0 to 24 by adding up the points from these prognostic predictors. Based on the ALBI-based BCLC system, patients with $\mathrm{ALBI}-\mathrm{BCLC}$ stage 0 had a nomogram score 0 . Patients with $\mathrm{ALBI}-\mathrm{BCLC}$ stage $A$ were assigned nomogram scores between 1.64 and 5.85; patients classified as ALBI-BCLC stage B had nomogram scores from 4.54 to 8.75 . Accordingly, patients with stage $C$ and $D$ were assigned nomogram scores of 2.78-16.99 and 6.66-23.53 respectively. As shown in Figure 2, the projections from total points on the scales below indicate the estimated survival probability at 3 and 5 years for study patients. 

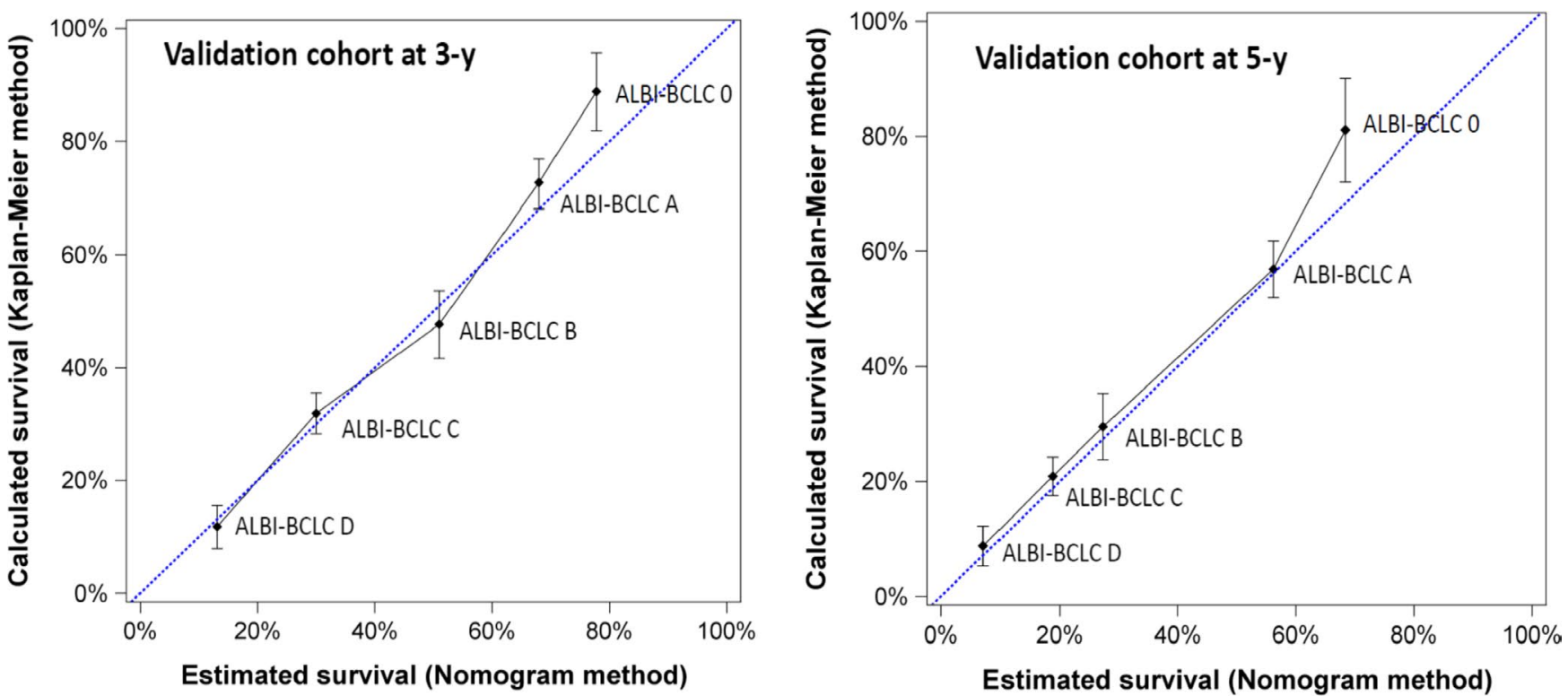

FIGURE 4 The calibration plots of the nomogram in the validation cohort for 3- and 5-y survival prediction. By dividing patients into five groups based on the nomogram points, the calibration plot excellently matched with the ideal 45 -degree reference line for survival prediction in patients with ALBI grade-based BCLC stages $A$ to $D$ at 3 and 5 y except for stage 0 patients. ALBI, albumin-bilirubin; BCLC, Barcelona Clinic Liver Cancer

\section{4 | Discrimination and calibration of nomogram in the derivation cohort}

The nomogram generated from the derivation cohort had a concordance index of 0.77 (95\% Cl: 0.71-0.81). In the calibration plot, the mean and $95 \% \mathrm{Cl}$ of survival rates calculated by the Kaplan-Meier method are shown on the $\mathrm{Y}$-axis, and the mean predicted survival rates estimated by the nomogram method are shown on the $X$-axis. The calibration plots for both 3- and 5-year survival well matched with the ideal 45-degree line for patients across ALBI-based BCLC 0 to ALBI-based BCLC D (Figure 3).

\subsection{Discrimination and calibration of nomogram in the validation cohort}

The nomogram had a concordance index 0.76 (95\% Cl: 0.71-0.81) in the validation cohort. In Figure 4, the calibration plot for 3- and 5 -year survival in the validation cohort well matched with the ideal 45-degree line for patients with ALBI-BCLC stages A to D. For stage 0 patients, the $95 \% \mathrm{Cl}$ of the survival estimated by the Kaplan-Meier method did not cover the nomogram-predicted survival.

\section{4 | DISCUSSION}

The BCLC staging system has been recommended as the practice guideline for HCC in both Europe and USA. ${ }^{3,4}$ However, a major shortcoming of the BCLC is that the prognosis of patients within the same cancer stage could greatly vary because of highly variable clinical presentations. This study adopted a more recently introduced ALBI grade as a marker of liver dysfunction to replace the traditional
CTP classification. With a large patient cohort and adequate followup, we have constructed and validated the new ALBI-BCLC system which may accurately predict the survival for HCC patients specifically at an individual level.

Using ALBI grade for risk stratification in HCC patients has a clear rationale. The ALBI grade is known to rely on only two single routine laboratory parameters which are inexpensive and objective. By contrast, the presence of ascites requires ultrasound confirmation, whilst the assessment of encephalopathy can be challenging and subjective in the CTP classification. In addition, the ALBI score is determined on the basis of mathematical calculation of continuous variables of serum albumin and bilirubin levels. However, the CTP classification uses prefixed cut-off points for objective variables including albumin, bilirubin and prothrombin time; these cut-offs could be arbitrary and patients at the extreme of distribution are classified equally as patients with marginally deranged laboratory parameters. Moreover, some of the variables considered in the CTP classification, such as ascites and serum albumin levels, are usually tightly interrelated. Notably, CTP classification is designed for patients with cirrhosis but many HCC patients may have normal or slightly derogated liver function. In this study, we confirm the feasibility of ALBI grade as an evidence-based measurement of liver functional reserve in $\mathrm{HCC}$ patients within the BCLC staging.

Tumour burden is a well-known prognostic predictor for HCC. In our study, tumour burden grade 3 shows the greatest impact on survival because it was associated with the highest BETA value in the Cox model. However, patients with tumour burden grade 3 are not considered as terminal stage (BCLC stage $D$ ) in the original CTP-based BCLC, in which CTP class C and PS 3-4 are required to define the terminal stage regardless of tumour burden. Our earlier study suggests that tumour burden was strongly associated with the outcome even 
in terminal stage patients. ${ }^{29}$ An example is that for patients with small tumour burden and CTP class C, they can still undergo RFA or liver transplantation to improve the overall survival. In the current study, survival differences were observed for patients within the same stage of BCLC because of variable tumour burden, liver functions and PS. Our findings show that about $33 \%$ of ALBI-based BCLC stage C and $15 \%$ of stage $D$ patients received curative treatments to prolong their survival. This may explain why there is a wide range of nomogram point for stage $C(2.78-16.99)$ and stage $D(6.66-23.53)$. Thus, the proposed ALBI-based nomogram for BCLC system is expected to more accurately estimate individual patient survival.

The concordance index in the derivation cohort was 0.77 , suggesting that the possibility of patients with smaller nomogram point having a longer survival is about $77 \%$ if patients with two different nomogram points are selected. In comparison with our previous study, ${ }^{23}$ the concordance indices of the derivation and validation sets were 0.76 and 0.77 , respectively, in the CTP-based BCLC nomogram. Therefore, the performance of the ALBI-based BCLC nomogram is considered as efficient as the CTP-based BCLC nomogram. Notably, the calibration plot in our study fell along with the ideal 45degree reference for different stages of ALBI-BCLC system in both patient cohorts, with the only exception of ALBI-BCLC stage 0 in the validation cohort. The relatively small sample size in this group may explain the cause of suboptimal performance in outcome prediction. Altogether, our data indicate that this ALBI-based nomogram of BCLC system could be an enhanced prognostic model for HCC.

Our approach has several clinical advantages. Firstly, this nomogram is an accurate and reproducible prognostic tool for individual HCC patient. Secondly, all the predictors included in the nomogram can be readily obtained in daily practice. Thirdly, this nomogram uses ALBI grade which is a more objective tool to assess liver dysfunction. Fourthly, this user-friendly nomogram may enable physicians to easily calculate survival risk at individual level. However, this study still has a few limitations. This nomogram was generated from a single centre where hepatitis B is the predominant aetiology of HCC; external validation is needed for countries where other aetiologies prevail. Also, our study included a small number of patients who received liver transplantation, and this nomogram might not be used in centres with a high volume of liver transplant. Lastly, the primary anticancer treatment was not included in the nomogram and further study is required to validate its prognostic performance.

In conclusion, the proposed ALBI-based nomogram of BCLC system for HCC is a feasible strategy in the precision medicine era. Our data indicate that it is a straightforward, reproducible and userfriendly prognostic tool to estimate the survival of individual HCC patient except for very early stage patients. Further studies are required to validate this new model to improve patient management.

\section{ACKNOWLEDGEMENT}

This study was supported by the grants from Taipei Veterans General Hospital (V107A-008, VN108-05, V108A-002, V108C-008), Taipei, Taiwan.

\section{CONFLICT OF INTEREST}

The authors do not have any disclosures to report.

\section{AUTHOR CONTRIBUTIONS}

Guarantor of the article: Teh-la Huo. S.-Y. Ho and T.-I. Huo performed the research and wrote the paper. C.-Y. Hsu, P.-H. Liu, C.-Y. Hsia, and C.-W. Su collected and analysed the data. H.-J. Lei, Y.-H. Huang, C.-C. Ko, R.-C. Lee, and M.-C. Hou contributed to study design and data collection. All authors approved the final version of the manuscript.

\section{ORCID}

Yi-Hsiang Huang (iD https://orcid.org/0000-0001-5241-5425

Chien-Wei Su (iD https://orcid.org/0000-0003-3889-7004

Teh-la Huo iD https://orcid.org/0000-0003-2202-521X

\section{REFERENCES}

1. Bray F, Ferlay J, Soerjomataram I, et al. Global cancer statistics 2018: GLOBOCAN estimates of incidence and mortality worldwide for 36 cancers in 185 countries. CA Cancer J Clin. 2018;68:394-424.

2. Liu PH, Hsu CY, Hsia CY, et al. Prognosis of hepatocellular carcinoma: assessment of eleven staging systems. J Hepatol. 2016;64:601-608.

3. EASL Clinical Practice Guidelines. Management of hepatocellular carcinoma. J Hepatol. 2018;69:182-236.

4. Villanueva A. Hepatocellular carcinoma. N Engl J Med. 2019;380:1450-1462

5. Johnson PJ, Berhane S, Kagebayashi C, et al. Assessment of liver function in patients with hepatocellular carcinoma: a new evidencebased approach-the ALBI grade. J Clin Oncol. 2015;33:550-558.

6. Liu $\mathrm{PH}, \mathrm{Hsu} \mathrm{CY}, \mathrm{Hsia} \mathrm{CY}$, et al. ALBI and PALBI grade predict survival for $\mathrm{HCC}$ across treatment modalities and BCLC stages in the MELD era. J Gastroenterol Hepatol. 2016;32:879-886.

7. Chan AW, Chong CC, Mo FK, et al. Incorporating albumin-bilirubin grade into the cancer of the liver Italian program system for hepatocellular carcinoma. J Gastroenterol Hepatol. 2017;32:221-228.

8. Chan AW, Kumada T, Toyoda $\mathrm{H}$, et al. Integration of albumin-bilirubin (ALBI) score into Barcelona Clinic Liver Cancer (BCLC) system for hepatocellular carcinoma. J Gastroenterol Hepatol. 2016;31:1300-1306.

9. Pinato DJ, Sharma R, Allara E, et al. The ALBI grade provides objective hepatic reserve estimation across each BCLC stage of hepatocellular carcinoma. J Hepatol. 2017;66:338-346.

10. Balachandran VP, Gonen M, Smith JJ, et al. Nomograms in oncology: more than meets the eye. Lancet Oncol. 2015;16:e173-180.

11. lasonos A, Schrag D, Raj GV, et al. How to build and interpret a nomogram for cancer prognosis. J Clin Oncol. 2008;26:1364-1370.

12. He SS, Wang CT, Peng ZW, et al. Development and external validation of a nomogram for predicting the overall survival of patients with stage II nasopharyngeal carcinoma after curative treatment. Cancer Manage Res. 2019;11:4403-4412.

13. Sadeghi M, Alamdaran SA, Daneshpajouhnejad P, et al. A logistic regression nomogram to predict axillary lymph node metastasis in early invasive breast cancer patients. Breast J. 2019;25(4):769-771. https://doi.org/10.1111/tbj.13340

14. Deng W, Zhang W, Yang J, et al. Nomogram to predict overall survival for thoracic esophageal squamous cell carcinoma patients 
after radical esophagectomy. Ann Surg Oncol. 2019;26:2890-2898. https://doi.org/10.1245/s10434-019-07393.

15. Hsu CY, Liu PH, Ho SY, et al. Using nomogram of the Barcelona Clinic Liver Cancer system for treatment selection in patients with stage $C$ hepatocellular carcinoma. BMC Cancer. 2018;18:289.

16. Zou Q, Li J, Wu D, et al. Nomograms for pre-operative and post-operative prediction of long-term survival of patients who underwent repeat hepatectomy for recurrent hepatocellular carcinoma. Ann Surg Oncol. 2016;23:2618-2626.

17. Forner A, Reig M, Bruix J. Hepatocellular carcinoma. Lancet. 2018;391:1301-1314.

18. Kao WY, Su CW, Chau GY, et al. A comparison of prognosis between patients with hepatitis $B$ and $C$ virus-related hepatocellular carcinoma undergoing resection surgery. World J Surg. 2011;35:858-867.

19. Grant BF, Dufour MC, Harford TC. Epidemiology of alcoholic liver disease. Semin Liver Dis. 1988;8:12-25.

20. Lee $\mathrm{YH}, \mathrm{Hsu} \mathrm{CY}$, Huang $\mathrm{YH}$, et al. Vascular invasion in hepatocellular carcinoma: prevalence, determinants and prognostic impact. J Clin Gastroenterol. 2014;48:734-741.

21. Hsu CY, Liu PH, Ho SY, et al. Metastasis in patients with hepatocellular carcinoma: prevalence, determinants, prognostic impact and ability to improve the Barcelona Clinic Liver Cancer system. Liver Int. 2018;38:1803-1811.

22. Ho SY, Liu PH, Hsu CY, et al. Comparison of twelve liver functional reserve models for outcome prediction in patients with hepatocellular carcinoma undergoing surgical resection. Sci Rep. 2018;8:4773.

23. Hsu CY, Liu PH, Hsia CY, et al. Nomogram of the Barcelona Clinic Liver Cancer system for individual prognostic prediction in hepatocellular carcinoma. Liver Int. 2016;36:1498-1506.
24. Oken MM, Creech RH, Tormey DC, et al. Toxicity and response criteria of the Eastern Cooperative Oncology Group. Am J Clin Oncol. 1982;5:649-655.

25. Huo TI, Huang YH, Wu JC, et al. Comparison of transarterial chemoembolization and percutaneous acetic acid injection as the primary loco-regional therapy for unresectable hepatocellular carcinoma: a prospective survey. Aliment Pharmacol Ther. 2004:19:1301-1308.

26. Huang $\mathrm{YH}$, Chen $\mathrm{CH}$, Chang TT, et al. The role of transcatheter arterial embolization in patients with resectable hepatocellular carcinoma: a nation-wide, multicenter study. Liver Int. 2004;24:419-424.

27. Liu $\mathrm{PH}, \mathrm{Hsu} \mathrm{CY}$, Hsia $\mathrm{CY}$, et al. Surgical resection versus radiofrequency ablation for single hepatocellular carcinoma $\leq 2 \mathrm{~cm}$ in a propensity score model. Ann Surg. 2016;263:538-545.

28. Harrell FE Jr, Lee KL, Mark DB. Multivariable prognostic models: issues in developing models, evaluating assumptions and adequacy, and measuring and reducing errors. Stat Med. 1996;15:361-387.

29. Hsu CY, Liu PH, Ho SY, et al. Impact of tumor burden on prognostic prediction for patients with terminal stage hepatocellular carcinoma: a nomogram study. PLoS ONE. 2017;12:e0188031.

How to cite this article: Ho S-Y, Hsu C-Y, Liu P-H, et al. Albumin-bilirubin grade-based nomogram of the BCLC system for personalized prognostic prediction in hepatocellular carcinoma. Liver Int. 2020;40:205-214. https://doi. org/10.1111/liv.14249 